\title{
Determination of rotations in three dimensions using two-dimensional portal image registration
}

\author{
Anthony E. Lujan, ${ }^{\text {a) }}$ James M. Balter, and Randall K. Ten Haken \\ Department of Nuclear Engineering and Radiological Sciences, Department of Radiation Oncology, \\ University of Michigan, Ann Arbor, Michigan 48109
}

(Received 4 March 1997; accepted for publication 19 February 1998)

\begin{abstract}
The relative relationships among anatomic features visualized on planar radiographic images change due to rotations of the patient out of the imaging plane. These changes can be predicted $a$ priori from a three-dimensional radiographic model of the patient. In this study we assess the feasibility of using that information together with a planar image feature alignment tool to account for out-of-plane rotations in the evaluation of subsequent clinical patient images. A series of digitally reconstructed radiographs (DRRs) with known patient rotations was generated from a computed tomography scan of an anthropomorphic head phantom. Fixed anatomic features were extracted, as seen in the DRRs of rotated anatomy and entered into a database. Alignment of features from test radiographs with those from an entry in this database yielded an estimate of rotation out of plane (database entry that resulted in the best fit via planar transformation) along with the planar components of setup errors in the rotated plane. Tests using DRRs and films show that it is possible to select anatomic features in AP skull radiographs with position and orientation sensitive to out-of-plane rotation. (c) 1998 American Association of Physicists in Medicine. [S0094-2405(98)00705-6]
\end{abstract}

Key words: image registration, portal imaging, patient setup uncertainty, treatment verification

\section{INTRODUCTION}

Setup errors are a primary concern in fractionated conformal radiotherapy. Radiographic images obtained on a semiregular basis (portal images) are routinely compared to simulation images to gauge the accuracy of patient setup. These comparisons are often purely qualitative. A variety of image alignment tools have recently been developed to help measure patient setup errors ${ }^{1-9}$ with reported accuracies of $2 \mathrm{~mm}$ or better. Most of these tools determine the coordinate transformation in the plane of projection that would most likely properly align the portal and simulation images. However, recent investigations indicate that rotations out of the plane of projection may change the spatial relationship of features used in the planar image alignment, resulting in erroneous estimations of the planar translations and rotations. ${ }^{10,11}$

A new class of alignment tools is currently under investigation to address this problem. Hanley et al. ${ }^{11}$ have developed a tool to quantitate rotations of the pelvis by measuring the relative change in position of anatomic features that are insensitive to small changes in rotation out of the plane. Gilhuijs et al. ${ }^{10}$ have developed a more complete tool for evaluating the head and neck setup position. Features representing bony ridges are extracted from a pair of portal images and backprojected through a computed tomography (CT) model of the patient for a series of test transformations to maximize the correspondence of the extracted features with bone.

These techniques rely on significant changes in the relative location of selected features in order to optimize the estimation of the out-of-plane rotation, however, it is clear from the inspection of radiographs that some features change location significantly under rotation and others do not. It is our goal to determine the feasibility of using anatomic features to determine rotations of the head from single or multiple radiographs using a planar image registration technique.

\section{METHODS AND MATERIALS}

This study used an Alderson Rando anthropomorphic head phantom (The Phantom Laboratory, Salem, NY). A CT image dataset at $3 \mathrm{~mm}$ slice thickness was obtained. These data were entered into the treatment planning system at the University of Michigan (UMPLAN, University of Michigan, Ann Arbor, MI) and an isocenter was established. Multiple digitally reconstructed radiographs (DRRs) ${ }^{12}$ were generated (a pixel size of 0.5479 pixels $/ \mathrm{mm}$ in the isocenter plane) for an Anterior-Posterior (AP) beam to simulate different rotations of the phantom about the AP, Left-Right (LR), and Superior-Inferior (SI) axes (the origin at the isocenter). Rotations about more than one axis were generated by applying the rotation about the original SI axis, followed by rotation about the rotated LR axis, and then rotation about the doubly rotated AP axis.

Megavoltage portal films of the same phantom for rotations about multiple axes were obtained on a racetrack microtron (Scanditronix Racetrack Microtron System MM50, Scanditronix AB, Upsala, Sweden). The gantry, collimator, and table angles were changed to simulate rotations of the phantom. A stereotactic frame (Radionics, Burlington, MA) was used to establish and maintain the phantom isocenter. Films $(10 \mathrm{MV})$ were taken with a $20 \times 20 \mathrm{~cm}^{2}$ square field and digitized using a laser film scanner (Lumisys, Inc.) (pixel size $=450 \mu$, spot size $=420 \mu$ ). The magnification and orientation of the digitized film images were scaled to the 


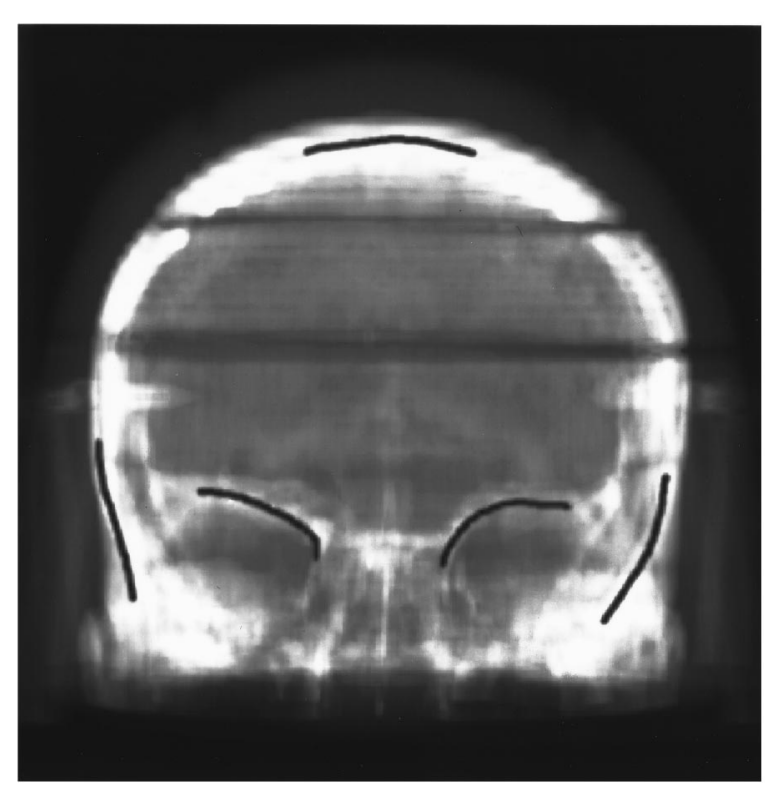

FIG. 1. Anatomy selected for contouring in an AP skull radiograph for use in image registration (see the text for a full description).

DRRs using the field borders as a reference. The digitized film images were then enhanced using unsharp masking and histogram equalization.

DRRs of rotated anatomy were visually inspected to find anatomy suitable for use in comparison to reference images through the use of an open curve matching algorithm. ${ }^{13}$ Useful anatomic features had to appear in each DRR, and the relative spatial relationships among these features had to change significantly under rotation (i.e., features sensitive to out-of-plane rotations). Anatomic features were selected so as not to extend along a single line or arc, thereby providing a unique planar transformation after alignment. The anatomy selected for this study include the outer table of the skull, the superior aspect of the right and left bony orbits, and the right and left petrous ridges as shown in Fig. 1.

The open curve matching algorithm finds the planar transformation (translations and rotation in the plane of projection) that results in a minimum root-mean-square (rms) residual distance value between points on user-defined curves on a reference image and those on a test image. When the test image is rotated out of the plane compared to the reference image, the rms residual distance will be larger than when the anatomy in the test and reference images lie in the same plane. This difference is a function of the image types of the reference and test images, the selected anatomic features, and the variation arising due to manual definition of the selected anatomy in both the reference and test image.

DRRs of rotated anatomy were aligned with a DRR representing the unrotated AP projection (the zero rotation DRR) to determine the ability of the algorithm to differentiate between small differences in rotation for the selected anatomic features for identical image types. Image alignment was repeated ten times (anatomy manually redefined in test and reference images) and the resultant rms residual distance was recorded for each alignment. The sensitivity of the algo- rithm was determined from these measurements by noting the magnitude of the out-of-plane rotation that resulted in a significantly different rms residual distance value when a DRR of rotated anatomy (test image) was aligned with the zero rotation DRR (reference image) compared to the rms residual distance value when a DRR is aligned with itself at a sensitivity of $P>95 \%$ (using a Student $t$ test of significance).

A library of DRRs representing AP projections with known rotations out of the plane was generated. Phantom rotations were simulated in $1.5^{\circ}$ steps from $-4.5^{\circ}$ to $4.5^{\circ}$ about each out-of-plane axis (LR, SI) and their combinations, resulting in 49 DRRs. This step size was chosen to cover the expected range of patient rotations as well as to provide good resolution based on the repeat alignment described above. The selected anatomy was defined manually on each DRR, and only the resultant contours were stored in a database for rapid access by the open curve matching algorithm. Thus, the resultant database contained 49 entries, where each entry consisted of the extracted contours and the out-of-plane rotation components corresponding to those contours. Another database of anatomy contour data was generated in $1^{\circ}$ steps from $-3^{\circ}$ to $3^{\circ}$ about the LR and SI axes in the same manner as the $1.5^{\circ}$ step size database to test the effect of step size on the estimate of the out-of-plane rotation components.

Digitized film images with known rotations and no translations were aligned using each database. Each reference database was used to estimate the rotation of the patient in three dimensions by comparison to a digitized test image as follows (Fig. 2):

(1) Selected anatomy contoured on a digitized test film (test contours).

(2) Resultant test contours aligned to each contour set in the reference database using the open curve matching algorithm.

(3) Planar transformation and the rms residual distance value recorded for each trial alignment.

(4) The minimum rms residual distance value identified.

(5) The database entry that corresponds to the minimum rms residual distance value gives the estimated magnitude of the out-of-plane rotation components, while the in-plane transformation is estimated by the corresponding planar transformation from the open curve matching algorithm.

To test the effect of image quality on the estimation of the out-of-plane rotation, DRRs with known rotations were aligned to the $1.5^{\circ}$ increment database. Finally, to determine the reproducibility of the image alignment arising from the variability in the manual contouring of the selected anatomy, several films and DRRs were contoured ten times by an experienced operator and aligned with the $1.5^{\circ}$ step size database.

\section{RESULTS}

Figure 3 shows the rms residual distance values as a function of the angle of rotation out of the plane when a film of anatomy with no rotation was aligned with the $1.5^{\circ}$ step size 
(a)

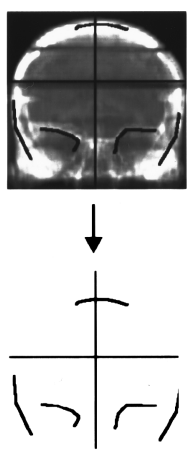

(b)

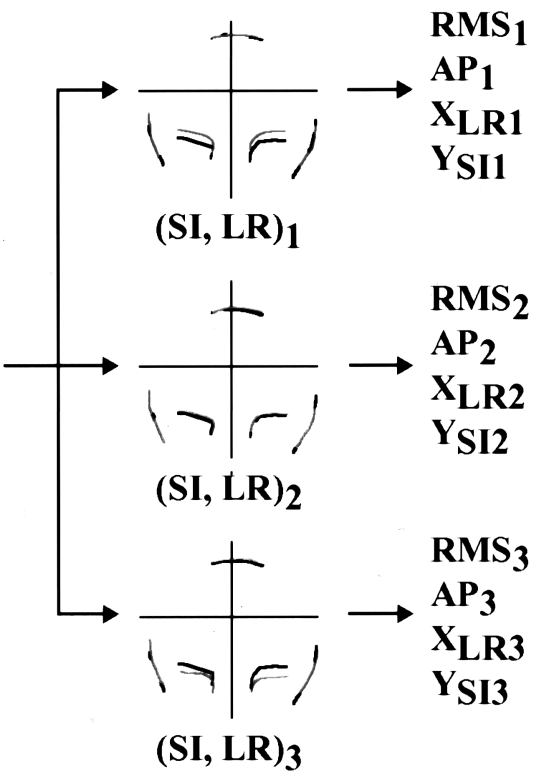

FIG. 2. The general procedure for estimating 3-D rotation of a patient using a reference database. (a) Selected anatomy contoured on a digitized film (test contours); (b) the resultant test contours aligned to each entry in the reference database; (c) each planar transformation and rms residual distance value was recorded and the minimum rms residual distance value was identified.

reference database. Repeated alignments of DRRs of rotated anatomy with the zero rotation DRR indicate the mean rms residual distance value increases as the rotation out of the plane increases. Further, it is noted that rms residual distance values from alignment with DRRs with rotations greater than $2^{\circ}$ out of the plane are significantly different $(P>95 \%)$ than

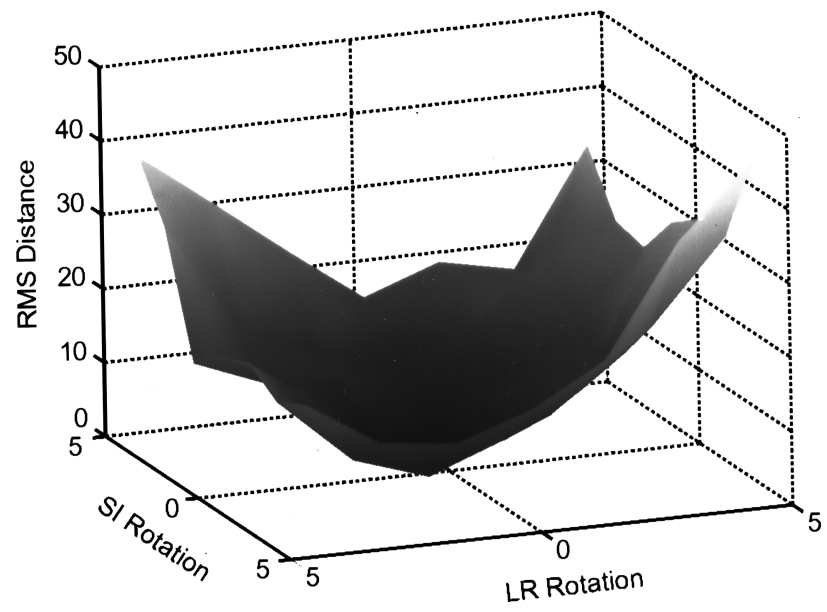

FIG. 3. The rms residual distance as a function of the angle of out-of-plane rotation for alignment of a portal image with no rotation to the $1.5^{\circ}$ increment database. the rms residual distance values when the zero rotation DRR is aligned to itself or to DRRs with rotations $1^{\circ}$ out of the plane.

Results from alignment of portal images with random rotations (in and out of plane) to the $1.5^{\circ}$ step size reference database are shown in Table I. The true (known) rotations about the AP, SI, and LR axis are shown, as well as the selected rotations about the SI and LR axes. The planar transformation (rotation about the AP axis and translations along the LR and SI axes) determined from the planar image alignment with the dataset that provided the selected out-ofplane rotation components is also shown. In most cases, the difference between the estimated and the true rotation is less than or equal to $2^{\circ}$. The in-plane (AP) rotation components in all cases are determined to within $2^{\circ}$ of the true rotation about the AP axis. The results from alignment of test images with the $1^{\circ}$ step size reference database (not shown) are consistent with the results shown in Table I. The differences between estimated and true rotations range up to $2^{\circ}$ about all axes.

The results of alignment of DRRs corresponding to the film images to the $1.5^{\circ}$ step size database are presented in Table II, and show that in all cases, the out-of-plane rotation is estimated to within $1.5^{\circ}$ (the resolution of the database). The in-plane rotation is determined in most cases to within $1^{\circ}$ (exceptions have at least one of the out-of-plane rotations outside the bounds of the database). The in-plane translations along both the SI and the LR axis are determined to less than $1.2 \mathrm{~mm}$, with one exception (which, again, is for a large out-of-plane rotation).

The results from repeat manual contouring of features seen in films and DRRs are shown in Tables III and IV. The average differences in rotation are shown in Table III, while the average translations are shown in Table IV. The repeat measurements indicate that there is a standard deviation in selecting the out-of-plane rotation of approximately $1^{\circ}$ about both the SI and LR axes for films and DRRs. The average difference between the selected rotation and the true rotation are less than $1^{\circ}$ for rotations about the LR and AP axes and less than $2^{\circ}$ for rotations about the SI axis. These results indicate the relative change in the anatomic features is smaller for rotations about the SI axis than for rotations about the LR or AP axes.

The average error in translation along the LR axis is less than $1 \mathrm{~mm}$, with a standard deviation for both DRRs and films of approximately $0.6 \mathrm{~mm}$. The average errors in translation along the SI axis for films range up to $2.4 \mathrm{~mm}$. The average error in translation along the SI axis from DRRs is less than $1 \mathrm{~mm}$. The standard deviation for both films and DRRs are similar. These measurements indicate there is a systematic error along the SI axis for the films of approximately $1.7 \mathrm{~mm}$. This results from an error in the placement of the isocenter along the SI axis.

\section{DISCUSSION}

We have described a method accounting for the out-ofplane rotation by using planar alignment to compare a portal 
TABLE I. Results from alignment of digitized films with a $1.5^{\circ}$ step reference database. The true rotations about the SI, LR, and AP axes are shown as well as the selected rotations out of the plane (about the SI and LR axes) and the in-plane rotation (AP axis) and the in-plane translations along the LR $\left(X_{\mathrm{LR}}\right)$ and SI $\left(Y_{\mathrm{SI}}\right)$ axes determined from the planar alignment with the dataset corresponding to the selected out-of-plane components.

\begin{tabular}{|c|c|c|c|c|c|c|c|}
\hline \multicolumn{6}{|c|}{ True and selected rotation (degrees) } & \multirow{2}{*}{\multicolumn{2}{|c|}{$\begin{array}{c}\text { In-plane } \\
\text { translation }(\mathrm{mm})\end{array}$}} \\
\hline \multicolumn{2}{|c|}{ Superior-Inferior } & \multicolumn{2}{|c|}{ Left-Right } & \multicolumn{2}{|c|}{ Anterior-Posterior } & & \\
\hline True & Selected & True & Selected & True & Selected & $X_{\mathrm{LR}}$ & $Y_{\mathrm{Si}}$ \\
\hline-3 & -3 & -1 & -3 & 2 & 0.3 & 0.6 & 0.6 \\
\hline-1 & 1.5 & -3 & -1.5 & 2 & 1.1 & 1.2 & 1.7 \\
\hline 1 & 1.5 & -2 & -1.5 & 0 & 1.3 & -1.2 & 2.9 \\
\hline 2 & 4.5 & -3 & -3 & 5 & 6.5 & 0.0 & 2.3 \\
\hline-2 & -1.5 & 1 & 1.5 & -2 & -2.8 & 0.0 & 1.2 \\
\hline 0 & 0 & 0 & -1.5 & 0 & 1.5 & 1.2 & 0.6 \\
\hline 3 & 3 & 0 & -1.5 & -2 & -1.2 & -1.2 & 1.2 \\
\hline 6 & 4.5 & -6 & -3 & 0 & 0.2 & 1.2 & 0.6 \\
\hline-4 & -3 & 3 & 3 & -5 & -6.9 & 1.7 & 1.7 \\
\hline 6 & 4.5 & -3 & -3 & 1 & 2.3 & -1.2 & 2.9 \\
\hline 5 & 4.5 & 0 & -1.5 & -5 & -6.9 & 1.2 & 1.7 \\
\hline
\end{tabular}

TABLE II. Results from alignment of DRRs with a $1.5^{\circ}$ step reference database. The true rotations about the SI, LR, and AP axes are shown as well as the selected rotations out of the plane (about the SI and LR axes) and the in-plane rotation (AP axis) and the in-plane translations along the $\mathrm{LR}\left(X_{\mathrm{LR}}\right)$ and $\mathrm{SI}\left(Y_{\mathrm{SI}}\right)$ axes determined from the planar alignment, with the dataset corresponding to the selected out-of-plane components.

\begin{tabular}{|c|c|c|c|c|c|c|c|}
\hline \multicolumn{6}{|c|}{ True and selected rotation (degrees) } & \multirow{2}{*}{\multicolumn{2}{|c|}{$\begin{array}{c}\text { In-plane } \\
\text { translation }(\mathrm{mm})\end{array}$}} \\
\hline \multicolumn{2}{|c|}{ Superior-Inferior } & \multicolumn{2}{|c|}{ Left-Right } & \multicolumn{2}{|c|}{ Anterior-Posterior } & & \\
\hline True & Selected & True & Selected & True & Selected & $X_{\mathrm{LR}}$ & $Y_{\mathrm{SI}}$ \\
\hline-3 & -4.5 & -1 & -1.5 & 2 & 1.8 & 0.6 & 0.0 \\
\hline-1 & 0 & -3 & -3 & 2 & 2.8 & 1.2 & -0.6 \\
\hline 1 & 1.5 & -2 & -1.5 & 0 & 0.2 & 0.0 & 0.0 \\
\hline 2 & 3 & -3 & -3 & 5 & 4.2 & 0.0 & 0.6 \\
\hline-2 & -1.5 & 1 & 1.5 & -2 & -1.2 & 0.0 & 0.0 \\
\hline 0 & 0 & 0 & 0 & 0 & 0.8 & -1.2 & 0.0 \\
\hline 3 & 1.5 & 0 & 0 & -2 & -2.3 & 0.6 & 0.0 \\
\hline 6 & 4.5 & -6 & -4.5 & 0 & 2.0 & 0.0 & 0.6 \\
\hline-4 & -3 & 3 & 4.5 & -5 & -3.6 & -1.2 & 0.0 \\
\hline 6 & 4.5 & -3 & -3 & 1 & -1.3 & 1.7 & 0.0 \\
\hline 5 & 4.5 & 0 & 0 & -5 & -6.9 & 0.6 & -1.2 \\
\hline
\end{tabular}

TABLE III. Average differences in rotation resulting from repeat contouring of films and DRRs.

\begin{tabular}{crrrllr}
\hline \hline $\begin{array}{c}\text { Image } \\
\text { type }\end{array}$ & $\mathrm{SI}_{t}$ & $\mathrm{LR}_{t}$ & $\mathrm{AP}_{t}$ & $\langle\delta \mathrm{SI}\rangle \pm \sigma$ & $\langle\delta \mathrm{LR}\rangle \pm \sigma$ & $\langle\delta \mathrm{AP}\rangle \pm \sigma$ \\
\hline FILM & 0 & 0 & 0 & $-0.8 \pm 0.7$ & $-0.6 \pm 1$ & $0.6 \pm 1.2$ \\
DRR & 0 & 0 & 0 & $-0.6 \pm 0.7$ & $0.2 \pm 0.5$ & $-0.6 \pm 1.2$ \\
FILM & 1 & -2 & 0 & $-1.3 \pm 0.7$ & -1 & Average difference in rotation (degrees) \\
DRR & 1 & -2 & 0 & $-0.5 \pm 0$ & $-0.5 \pm 0$ & $-0.9 \pm 1.5$ \\
FILM & -2 & 1 & -2 & $-1.6 \pm 0.6$ & $-0.4 \pm 0.7$ & $-0.5 \pm 0.8$ \\
FILM & -3 & -1 & 2 & $-0.9 \pm 1.4$ & $-0.1 \pm 1.2$ & $-0.3 \pm 1.3$ \\
\hline \hline
\end{tabular}


TABLE IV. Average magnitude of translations determined from repeat contouring of films and DRRs.

\begin{tabular}{crrrrrr}
\hline \hline \multirow{2}{*}{$\begin{array}{c}\text { Image } \\
\text { type }\end{array}$} & \multicolumn{3}{c}{ True rotation } & & \multicolumn{2}{c}{ Average translations $(\mathrm{mm})$} \\
\cline { 2 - 3 } \cline { 6 - 6 } \cline { 5 - 6 } FILM & SI & LR & AP & & $\left\langle X_{\text {LR }}\right\rangle \pm \sigma$ & $\left\langle Y_{\text {SI }}\right\rangle \pm \sigma$ \\
DRR & 0 & 0 & 0 & & $0.7 \pm 0.6$ & $1.9 \pm 0.5$ \\
FILM & 0 & 0 & 0 & & $-0.3 \pm 0.8$ & $-0.3 \pm 0.5$ \\
DRR & 1 & -2 & 0 & & $-0.1 \pm 0.6$ & $2.4 \pm 0.7$ \\
FILM & -2 & -2 & 0 & & $-0.1 \pm 0.7$ & $-0.2 \pm 0.7$ \\
FILM & -3 & 1 & -2 & & $0.4 \pm 0.4$ & $1.7 \pm 0.7$ \\
& -1 & 2 & $0.4 \pm 0.6$ & $1.4 \pm 0.6$ \\
\hline \hline
\end{tabular}

image with a database of contours representing rotated anatomy. The estimate for the out-of-plane rotation components is selected from one of the discrete members of a database, while the in-plane rotation component and the inplane translations are given from the alignment of the test image with the database entry that corresponds to the selected out-of-plane rotation components.

In most cases, the selected rotation is within one entry in the database from the optimal selection. Thus, it is clear that the accuracy of the method is a function of the step size of the database. However, measurements made using a finer step size $\left(1^{\circ}\right.$ vs $\left.1.5^{\circ}\right)$ do not indicate an increased accuracy in the selection of the out-of-plane rotation in many cases. These results are not inconsistent, as the smaller step size is below the accuracy limits of the algorithm based on the selected anatomy, and are supported by the measurements that indicate that the rms residual distance values are similar for differences in rotation of less than $2^{\circ}$. Surface plots of the rms residual distance as a function of the out-of-plane angle of rotation for random rotations show that the rms residual distance increases as the difference between the true rotation and the test rotation increases, however, for test rotations near the true rotation the rms residual distance values are very similar.

Alignment of DRRs with the $1.5^{\circ}$ step size reference database indicate more accurate and reproducible estimates of the out-of-plane rotation components as well as the in-plane rotation and translations than alignment of film. The anatomy of interest is more clearly seen on a DRR versus a megavoltage film, which leads to the improved accuracy and reproducibility of the estimate. These results stress the importance of high quality portal and reference images.

Again, for our discrete system, the minimum rms residual value selects the out-of-plane rotation components, however, the true minimum may not lie at the selected point. As an extension of this work, it should be possible to fit a function to the rms residual distance value surface contours and mathematically determine the minimum rms residual distance value. This may give a more accurate answer than simply choosing the discrete minimum value to select the rotation.

The accuracy of the three-dimensional (3-D) alignment may also be improved by correlating information from orthogonal radiographs. Lateral films of skull anatomy were obtained and visually inspected, but it was not possible to select anatomic features in a lateral view that satisfied the criteria necessary for use with the open curve matching al- gorithm. Most notably, it was difficult to find a consistent set of features whose relative position was obviously changed under rotation. However, using projection angles different than AP and lateral angles may reveal suitable anatomy.

The accuracy of the current method, however, could allow for an increase in the overall precision of patient setup. As noted by Hanley, ${ }^{11}$ rotations more than $2^{\circ}$ out of the imaging plane can result in dosimetrically significant consequences when simple planar alignment is used to verify and correct for patient position for treatment of the prostate. One could expect changes of the same order to be as or more important in treatments of the head and neck due to the smaller separation of the anatomy in this site. This method provides 3-D alignment information with a minimal amount of time at the treatment machine (the majority of the time is spent computing the DRRs in advance of the first day of treatment).

A minor drawback to using this method for image alignment is that multiple DRRs must be computed a priori. This can be time intensive both from a computational as well as a personnel standpoint. However, there have been methods proposed for quickly computing DRRs and extracting anatomic information $^{14,10}$ to provide information on the threedimensional patient transformatio

\section{ACKNOWLEDGMENTS}

This work was supported by NIH Grant No. P01CA59827.

${ }^{a}$ Electronic mail: aelujan@umich.edu

${ }^{1}$ J. Bijhold, K. G. A. Gilhuijs, M. van Herk, and H. Meertens, "Radiation field edge detection in portal images," Phys. Med. Biol. 12, 1705-1710 (1991).

${ }^{2}$ K. G. A. Gilhuijs, A. A. H. El-Gayed, M. van Herk, and R. E. Vijlbrief, "An algorithm for automatic analysis of portal images: Clinical evaluation for prostate treatments," Radiother. Oncol. 29, 261-268 (1993).

${ }^{3}$ K. G. A. Gilhuijs, A. Touw, M. van Herk, and R. E. Vijlbrief, "Optimization of automatic portal image analysis," Med. Phys. 22, 1089-1099 (1995).

${ }^{4}$ K. G. A. Gilhuijs and M. van Herk, "Automatic on-line inspection of patient setup in radiation therapy using digital portal images," Med. Phys. 20, 667-677 (1993).

${ }^{5}$ M. G. Herman, R. A. Abrams, and R. R. Mayer, "Clinical use of on-line portal imaging for daily patient treatment verification," Int. J. Radiat. Oncol., Biol., Phys. 28, 1017-1023 (1994).

${ }^{6}$ R.-D. Kortmann, C. F. Hess, R. Jany, C. Meisner, and M. Bamberg, "Reproducibility of field alignment in difficult patient positioning," Int. J. Radiat. Oncol., Biol., Phys. 29, 869-872 (1994).

${ }^{7}$ J. M. Michalski, J. W. Wong, W. R. Bosch, D. Yan, A. Cheng, R. L. Gerber, M. V. Graham, D. Low, R. K. Valicenti, and J. V. Piephoff, "An evaluation of two methods of anatomical alignment of radiotherapy portal 
images,' Int. J. Radiat. Oncol., Biol., Phys. 27, 1199-1206 (1993).

${ }^{8}$ M. J. Murphy, "An automatic six-degree-of-freedom image registration algorithm for image-guided frameless stereotaxic radiosurgery," Med. Phys. 26, 857-66 (1997).

${ }^{9}$ J. Wong, D. Yan, J. Michalski, M. Graham, K. Halverson, W. Harms, and J. Purdy, "The cumulative verification image analysis tool for offline evaluation of portal images," Int. J. Radiat. Oncol., Biol., Phys. 33, 1301-1310 (1995).

${ }^{10}$ K. G. A. Gilhuijs, K. Drukker, A. Touw, P. J. van de Ven, and M. van Herk, "Interactive three dimensional inspection of patient setup in radiation therapy using digital portal images and computed tomography data," Int. J. Radiat. Oncol., Biol., Phys. 34, 873-885 (1996).

${ }^{11}$ J. Hanley, G. S. Mageras, J. Sun, and G. J. Kutcher, "The effects of out-of-plane rotations on two dimensional portal image registration in conformal radiotherapy of the prostate," Int. J. Radiat. Oncol., Biol., Phys. 33, 1331-1343 (1995).

${ }^{12} \mathrm{G}$. W. Sherouse, K. Novine, and E. L. Chaney, "Computation of digitally reconstructed radiographs for use in radiotherapy treatment design," Int. J. Radiat. Oncol., Biol., Phys. 18, 651-658 (1990).

${ }^{13}$ J. M. Balter, C. A. Pelizzari, and G. T. Y. Chen, "Correlation of projection radiographs in radiation therapy using open curve segments and points," Med. Phys. 19, 329-334 (1992).

${ }^{14}$ D. S. Fritsch, E. L. Chaney, A. Boxwala, M. J. McAuliffe, S. Raghavan, A. Thall, and J. R. D. Earnhart, "Core-based portal image registration for automatic radiotherapy treatment verification," Int. J. Radiat. Oncol., Biol., Phys. 33, 1287-1300 (1995) 